International Journal of Pure and Applied Mathematics

Volume $91 \quad$ No. 2 2014, 197-207

ISSN: 1311-8080 (printed version); ISSN: 1314-3395 (on-line version)

url: http://www.ijpam.eu

doi: http://dx.doi.org/10.12732/ijpam.v91i2.6

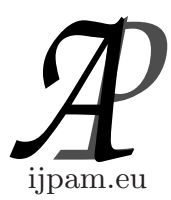

\title{
MORPHISMS ON CLOSURE SPACES AND MOORE SPACES
}

\author{
B. Venkateswarlu ${ }^{1}$, R. Vasu Babu ${ }^{2}$, Getnet Alemu ${ }^{3}$ \\ ${ }^{1}$ Department of Mathematics \\ GIT, GITAM University \\ Visakhapatnam, 530 045, A.P., INDIA \\ ${ }^{2}$ Department of Mathematics \\ Shri Vishnu Engineering college for Women \\ Bhimavaram, 534201, W.G. Dist., A.P., INDIA \\ ${ }^{3}$ Department of Mathematics \\ Gondar University \\ Gondar, ETHIOPIA
}

\begin{abstract}
We discuss an equivalence of the concepts of complete lattices, Moore classes, and a one-to-one correspondence between Moore classes of subsets of a set $X$ and closure operators on $X$. Also, we establish a correspondence between closure operators on sets and complete lattices. We describe morphisms among partially ordered sets, lattices, Moore classes, closure operators and complete lattices and discuss certain inter-relationships between these objects.
\end{abstract}

AMS Subject Classification: 06A15, 06F30, 54H12

Key Words: closure operator, Moore class, compact element, complete lattice, algebraic Lattice, frame, morphisms

\section{Introduction and Preliminaries}

A partially ordered set (poset) is a pair $(X, \leq)$, where $X$ is a non empty set and

Received: September 26, 2013

(c) 2014 Academic Publications, Ltd. url: www.acadpubl.eu

$\S_{\text {Correspondence author }}$ 
$\leq$ is a partial order (a reflexive, transitive and antisymmetric binary relation) on $X$. For any subset $A$ of $X$ and $x \in X, x$ is called a lower bound (upper bound) of $A$ if $x \leq a$ ( $a \leq x$ respectively) for all $a \in A$. A poset $(X, \leq)$ is called a lattice if every nonempty finite subset of $X$ has greatest lower bound ( or glb or infimum ) and least upper bound ( or lub or supremum) in $X$. If $(X, \leq)$ is a lattice and, for any $a, b \in X$, if we define $a \wedge b=$ infimum $\{a, b\}$ and $a \vee b=$ supremum $\{a, b\}$, then $\wedge$ and $\vee$ are binary operations on $X$ which are commutative, associative and idempotent and satisfy the absorption laws $a \wedge(a \vee b)=a=a \vee(a \wedge b)$. Conversely, any algebraic system $(X, \wedge, \vee)$ satisfying the above properties becomes a lattice in which the partial order is defined by $a \leq b \Longleftrightarrow a=a \wedge b \Longleftrightarrow a \vee b=b$. A lattice $(X, \wedge, \vee)$ is called distributive if $a \wedge(b \vee c)=(a \wedge b) \vee(a \wedge c)$ for all $a, b, c \in X$ ( equivalently $a \vee(b \wedge c)=(a \vee b) \wedge(a \vee c)$ for all $a, b, c \in X)$. A lattice $(X, \wedge, \vee)$ is called a bounded lattice if it has the smallest element 0 and largest element 1 ; that is, there are elements 0 and 1 in $X$, such that $0 \leq x \leq 1$ for all $x \in X$.

A partially ordered set in which every subset has infimum and supremum is called a complete lattice. If $(L, \leq)$ is a complete lattice and $X \subseteq L$, we write $\inf X$ or $\wedge X$ or $\bigwedge_{x \in X} x$ for the infimum of $X$ and $\sup X$ or $\vee X$ or $\bigvee_{x \in X} x$ for the supremum of $X$. If $X=\left\{x_{1}, x_{2}, \cdots, x_{n}\right\}$ is a finite subset, then we write $\bigwedge_{i=1}^{n} x_{i}$ or $x_{1} \wedge x_{2} \wedge \cdots \wedge x_{n}$ for $\inf X$ and $\bigvee_{i=1}^{n} x_{i}$ or $x_{1} \vee x_{2} \vee \cdots \vee x_{n}$ for $\sup X$. Any complete lattice has the smallest element and the greatest element which are denoted by 0 and 1 respectively.Logically, the infimum and supremum of the empty set are 1 and 0 respectively. An element $a \neq 0$ in a complete lattice $L$ is called compact if, for any $A \subseteq L, a \leq \sup A \Longrightarrow a \leq \sup F$ for some finite $F \subseteq A$. A complete lattice in which every element is the supremum of a set of compact elements is called an algebraic lattice. For elementary properties of posets and lattices we refer to [1] and [2].

\section{Moore Classes and Closure Operators}

In this section, we introduce the notion of a Moore class and discuss certain important elementary properties of these. To begin with, we have the following.

Definition 2.1. Let $X$ be any non-empty set. A non-empty class $\mathscr{M}$ of subsets of $X$ is called a Moore class on $X$ if $\mathscr{M}$ is closed under arbitrary intersections, in the sense that, if $\left\{M_{\alpha}\right\}_{\alpha \in \Delta}$ is a subclass of $\mathscr{M}$, then $\bigcap_{\alpha \in \Delta} M_{\alpha} \in$ 
$\mathscr{M}$.

Example 2.2. Let $X$ be a topological space and $\mathscr{C}$ be the class of all closed subsets of $X[3]$. Then $\mathscr{C}$ is a Moore class on the set $X$.

Example 2.3. Let $G$ be a group and $\mathscr{S}$ be the class of all subgroups of $G$. Then $\mathscr{S}$ is a Moore class on the set $G$.

Example 2.4. Let $A$ be any universal algebra [ 4 ], where there is atleast one fundamental nullary operation, and let $\mathscr{S}$ be the class of all subalgebras of $A$. If $a_{0}$ is an element of $A$ corresponding to a fundamental nullary operation on $A$, then $a_{0}$ belongs to every subalgebra of $A$. From this it follows that the intersection of any class of subalgebras of $A$ is non-empty and hence a subalgebra of $A$. Thus $\mathscr{S}$ is a Moore class on $A$.

Example 2.5. For any non-empty set $X$, the whole power set $\mathscr{P}(X)$ is a Moore class on $X$ and is called the discrete Moore class on $X$.

Example 2.6. Let $X$ be a non-empty set and $A \subseteq X$. Then the class $\{A, X\}$ is a Moore class on $X$.

Since the intersection of the empty class of subsets of a set $X$ is the whole set $X$, it follows that any Moore class necessarily contains $X$. The following is a straight forward verification.

Theorem 2.7. Let $\mathscr{M}$ be a Moore class on a set $X$. For any subset $A$ of $X$, define

$$
\bar{A}=\cap\{M \in \mathscr{M} \mid A \subseteq M\} .
$$

Then the following hold for any subsets $A$ and $B$ of $X$

(1). $A \subseteq \bar{A}$

(2). $\overline{\bar{A}}=\bar{A}$

(3). $A \subseteq B \Longrightarrow \bar{A} \subseteq \bar{B}$

(4). $\bar{A}$ is the smallest member in $\mathscr{M}$ containing $A$.

Corollary 2.8. If $\mathscr{M}$ is a Moore class on $X$, then $\mathscr{M}=\{A \subseteq X \mid$ $\bar{A}=A\}$.

Note that in 2.2 above, $\bar{A}$ is the topological closure of $A$, while in $2.3, \bar{A}$ is the subgroups generated by $A$.

Definition 2.9. Let $X$ be a non-empty set and $\mathscr{P}(X)$ the set of all subsets of $X$. A mapping $c: \mathscr{P}(X) \rightarrow \mathscr{P}(X)$ is called a closure operator on $X$ if it satisfies the following for any $A$ and $B$ in $\mathscr{P}(X)$ 
(1). $c$ is extensive ; that is, $A \subseteq c(A)$

(2). $c$ is idempotent; that is, $c(c(A))=c(A)$

(3). $c$ is inclusion preserving; that is, $A \subseteq B \Rightarrow c(A) \subseteq c(B)$.

The following is an immediate consequence of Theorem 2.7.

Theorem 2.10. Let $\mathscr{M}$ be a Moore class on a set $X$ and, for any $A \subseteq X$, define

$$
c_{\mathscr{M}}(A)=\cap\{M \in \mathscr{M} \mid A \subseteq M\} .
$$

Then $c_{\mathscr{M}}$ is a closure operator on $X$.

The following is routine verification.

Theorem 2.11. Let $c$ be a closure operator on a set $X$ and

$$
\mathscr{M}_{c}=\{A \subseteq X \mid c(A)=A\}=\{c(A) \mid A \subseteq X\}
$$

Then $\mathscr{M}_{c}$ is a Moore class on $X$. Also, $c \mapsto \mathscr{M}_{c}$ is a one-to-one correspondence between the closure operators on $X$ and Moore classes on $X$.

Definition 2.12. A closure operator $c$ on a set $X$ is called a topological closure operator if $c(\phi)=\phi$ and $c(A \cup B)=c(A) \cup c(B)$ for any subsets $A$ and $B$ of $X$.

Theorem 2.13. Let $c$ be a closure operator on a set $X$ and $\mathscr{M}_{c}$ be the Moore class on $X$ corresponding to $c$, as given in Theorem 2.11. Then $c$ is a topological closure operator on $X$ if and only if $\mathscr{M}_{c}$ is closed under finite unions, in the sense that, for any finite subclass $\left\{M_{i}\right\}_{i \in I}$ of $\mathscr{M}_{c}, \bigcup_{i \in I} M_{i} \in \mathscr{M}_{c}$.

Proof. Suppose that $c$ is a topological closure operator on $X$. First, let us observe that the empty set $\phi$ belongs to $\mathscr{M}_{c}$, since $c(\phi)=\phi$. Let $\left\{M_{i}\right\}_{i \in I}$ be a finite subclass of $\mathscr{M}_{c}$. If $I$ is empty, then

$$
\bigcup_{i \in I} M_{i}=\phi \in \mathscr{M}_{c}
$$

Therefore, we can assume that $I$ is non-empty, say $I=\{1,2, \ldots, n\}$, where $n$ is a positive integer. Then

$$
\begin{aligned}
\bigcup_{i \in I} M_{i} & =M_{1} \cup M_{2} \cup \ldots \cup M_{n} \\
& =c\left(M_{1}\right) \cup c\left(M_{2}\right) \cup \ldots \cup c\left(M_{n}\right), \text { since } M_{i} \in \mathscr{M}_{c}
\end{aligned}
$$




$$
=c\left(M_{1} \cup M_{2} \cup \ldots \cup M_{n}\right)
$$

and therefore $\bigcup_{i \in I} M_{i} \in \mathscr{M}_{c}$. Thus $\mathscr{M}_{c}$ is closed under finite unions.

Conversely, suppose that $\mathscr{M}_{c}$ is closed under finite unions. In particular, $\phi \in \mathscr{M}_{c}$, since the union of the empty class of sets is empty. Therefore $c(\phi)=\phi$. Also, for any subsets $A$ and $B$ of $X$, we have

$$
c(c(A))=c(A) \text { and } c(c(B))=c(B)
$$

and hence $c(A)$ and $c(B)$ are members of $\mathscr{M}_{c}$. Since $\mathscr{M}_{c}$ is closed under finite unions, it follow that

$$
c(A) \cup c(B) \in \mathscr{M}_{c}
$$

and therefore $c(c(A) \cup c(B))=c(A) \cup c(B)$.

$$
\text { Now, } \begin{aligned}
c(A) \cup c(B) & \subseteq c(A \cup B) \\
& \subseteq c(c(A) \cup c(B)) \\
& =c(A) \cup c(B)
\end{aligned}
$$

and hence $c(A \cup B)=c(A) \cup c(B)$. Thus $c$ is a topological closure operator.

The following can be easily verified.

Theorem 2.14. Let $c$ be a closure operator on a set $X$. Then $c$ is topological if and only if there is a unique topology on $X$ with respect to which $c(A)$ is the closure of $A$, for all subsets $A$ of $X$.

\section{Complete Lattices}

In this section, we establish a correspondence between Moore classes on sets and complete lattices. Let us first recall that two posets $(X, \leq)$ and $(Y, \leq)$ are said to be isomorphic if there is a bijection $f: X \rightarrow Y$ such that $a \leq b$ in $X \Leftrightarrow f(a) \leq f(b)$ in $Y$. If $\mathscr{M}$ is a Moore class on a set $X$, then $\mathscr{M}$ becomes a complete lattice under the set inclusion ordering in which, for any $\left\{M_{i}\right\}_{i \in I}$ in $\mathscr{M}$,

$$
\inf \left\{M_{i}\right\}_{i \in I}=\bigcap_{i \in I} M_{i} \text { and } \sup \left\{M_{i}\right\}_{i \in I}=\overline{\bigcup_{i \in I} M_{i}}
$$

The converse of this is proved in the following. 
Theorem 3.1. Let $(L, \leq)$ be a complete lattice. Then $(L, \leq)$ is isomorphic to the Moore class $\mathscr{M}_{c}$ corresponding to a closure operator $c$ on a suitable set.

Proof. Consider the set $L$ and define,

$$
c: \mathscr{P}(L) \longrightarrow \mathscr{P}(L) \text { by } c(A)=\{x \in L \mid x \leq \sup A\}
$$

for any subset $A$ of $L$. Then, clearly $A \subseteq c(A)$ and

$$
A \subseteq B \Longrightarrow \sup A \leq \sup B \Longrightarrow c(A) \subseteq c(B)
$$

for any $A$ and $B$ in $\mathscr{P}(L)$. Also, for any $A \subseteq L$,

$$
\sup A \in c(A) \text { and } x \leq \sup A \text { for all } x \in c(A)
$$

and hence $\sup A=\sup c(A)$, so that $c(c(A))=c(A)$. Thus $c$ is a closure operator on the set $L$. Now consider the Moore class $\mathscr{M}_{c}$ defined by

$$
\mathscr{M}_{c}=\{A \subseteq X \mid c(A)=A\} .
$$

For any $a \in L$, let $c(a)$ denote $c(\{a\})$. Define

$$
f: L \longrightarrow \mathscr{M}_{c} \text { by } f(a)=c(a) \text { for any } a \in L .
$$

Clearly, $a \leq b \Longleftrightarrow f(a) \subseteq f(b)$ for all $a, b \in L$. This implies that $f$ is an injection also. Further, for any $A \in \mathscr{M}_{c}$, we have $A=c(A)=f(\sup A)$. Therefore $f$ is a surjection too. Thus $f: L \longrightarrow \mathscr{M}_{c}$ is an isomorphism.

\section{Morphisms}

In this section, we describe morphisms among partially ordered sets, lattices, Moore classes, closure operators and complete lattices and discuss certain interrelationships between these. First we recall the following.

Definition 4.1. Let $\left(X_{1}, \leq\right)$ and $\left(X_{2}, \leq\right)$ be partially ordered sets. A mapping $f: X_{1} \longrightarrow X_{2}$ is called an order-preserving mapping or an order homomorphism, if

$$
a \leq b \text { in } X_{1} \Longrightarrow f(a) \leq f(b) \text { in } X_{2}
$$

for any $a$ and $b \in X_{1}$. A bijection $f: X_{1} \longrightarrow X_{2}$ is called an order isomorphism if

$$
a \leq b \text { in } X_{1} \Longleftrightarrow f(a) \leq f(b) \text { in } X_{2}
$$

for any $a$ and $b$ in $X_{1}$. 
Definition 4.2. Let $\left(X_{1}, \wedge, \vee\right)$ and $\left(X_{2}, \wedge, \vee\right)$ be lattices and $f: X_{1} \longrightarrow$ $X_{2}$ be a mapping. Then

(1). $f$ is called a meet homomorphism if $f(a \wedge b)=f(a) \wedge f(b)$ for all $a, b \in$ $X_{1}$.

(2). $f$ is called a join homomorphism if $f(a \vee b)=f(a) \vee f(b)$ for all $a, b \in X_{1}$.

(3). $f$ is called a lattice homomorphism if $f$ is both a meet and join homomorphism.

(4). $f$ is called a meet ( or join or lattice) isomorphism if $f$ is a bijection and meet ( respectively join or lattice) homomorphism.

Remark 4.3. For any $f: X_{1} \rightarrow X_{2}$, it is clear that (3) implies (1) or (2) and that either of (1) or (2) implies that $f$ is an order homomorphism. One can construct examples of lattices to establish that the converse of each of these implications is not true.

Definition 4.4. A pair $(X, \mathscr{M})$ is called a Moore space if $X$ is a non-empty set and $\mathscr{M}$ is a Moore class on $X$.

Definition 4.5. A pair $(X, c)$ is called a closure space if $X$ is a non-empty set and $c$ is a closure operator on $X$.

Definition 4.6. Let $\left(X_{1}, \mathscr{M}_{1}\right)$ and $\left(X_{2}, \mathscr{M}_{2}\right)$ be Moore spaces. A mapping $f: X_{1} \longrightarrow X_{2}$ is called a homomorphism of Moore spaces if $f^{-1}(A) \in \mathscr{M}_{1}$ for all $A \in \mathscr{M}_{2}$.

Definition 4.7. Let $\left(X_{1}, c_{1}\right)$ and $\left(X_{2}, c_{2}\right)$ be closure spaces and $f: X_{1} \longrightarrow$ $X_{2}$ a mapping. Then $f$ is said to be a homomorphism of closure spaces if

$$
f\left(c_{1}(A)\right) \subseteq c_{2}(f(A)) \text { for all } A \subseteq X_{1} .
$$

The following result describes the inter relationships between homomorphisms of Moore spaces and those of closure spaces. First, recall from 2.10 that a Moore space $(X, \mathscr{M})$ induces a closure operator $c_{\mathscr{M}}$ on $X$ where $c_{\mathscr{M}}$ is defined by

$$
c_{\mathscr{M}}(A)=\cap\{M \in \mathscr{M} \mid A \subseteq M\} \text {, for any } A \subseteq X .
$$

Theorem 4.8. Let $\left(X_{1}, \mathscr{M}_{1}\right)$ and $\left(X_{2}, \mathscr{M}_{2}\right)$ be Moore spaces and $\left(X_{1}, c_{\mathscr{M}_{1}}\right)$ and $\left(X_{2}, c_{\mathscr{N}_{2}}\right)$ be the corresponding closure spaces. Then any mapping $f$ : $X_{1} \longrightarrow X_{2}$ is a homomorphism of Moore spaces if and only if it is a homomorphism of closure spaces. 
Proof. For simplicity, Let us write $c_{1}$ and $c_{2}$ for $c_{\mathscr{M}_{1}}$ and $c_{\mathscr{M}_{2}}$ respectively. Let $f: X_{1} \longrightarrow X_{2}$ be a mapping. First suppose that $f$ is a homomorphism of Moore spaces; that is,

$$
f^{-1}(M) \in \mathscr{M}_{1} \quad \text { for all } \quad M \in \mathscr{M}_{2} .
$$

Now, for any subset $A$ of $X_{1}$ and $M \in \mathscr{M}_{2}$, we have

$$
\begin{aligned}
f(A) \subseteq M & \Longrightarrow A \subseteq f^{-1}(f(A)) \subseteq f^{-1}(M) \in \mathscr{M}_{1} \\
& \Longrightarrow c_{1}(A) \subseteq f^{-1}(M) \\
& \Longrightarrow f\left(c_{1}(A)\right) \subseteq f\left(f^{-1}(M)\right) \subseteq M .
\end{aligned}
$$

From this it follows that $f\left(c_{1}(A)\right) \subseteq \cap\left\{M \in \mathscr{M}_{2} \mid f(A) \subseteq M\right\}=c_{2}(f(A))$. Thus $f: X_{1} \longrightarrow X_{2}$ is a homomorphism of closure spaces.

Conversely, suppose that $f$ is a homomorphism of closure spaces; that is,

$$
f\left(c_{1}(A)\right) \subseteq c_{2}(f(A)) \text { for all } A \subseteq X .
$$

For any $M \in \mathscr{M}_{2}$, we have

$$
\begin{aligned}
f\left(c_{1}\left(f^{-1}(M)\right)\right) & \subseteq c_{2}\left(f\left(f^{-1}(M)\right)\right) \\
& \subseteq c_{2}(M), \quad \text { Since } f\left(f^{-1}(M)\right) \subseteq M \\
& =M, \text { since } M \in \mathscr{M}_{2} .
\end{aligned}
$$

So that $c_{1}\left(f^{-1}(M)\right) \subseteq f^{-1}(M)$ and therefore $f^{-1}(M)=c_{1}\left(f^{-1}(M)\right) \in \mathscr{M}_{1}$. Therefore $f$ is a homomorphism of Moore spaces.

Definition 4.9. Let $\left(L_{1}, \leq\right)$ and $\left(L_{2}, \leq\right)$ be complete lattices. A function $f: L_{1} \longrightarrow L_{2}$ is called complete join homomorphism if

$$
f(\sup A)=\sup f(A) \text { for all } A \subseteq L_{1}
$$

$f$ is called a complete meet homomorphism if

$$
f(\inf A)=\inf f(A), \text { for all } A \subseteq L_{1} .
$$

Clearly every complete join or meet homomorphism is an order homomorphism. Also, any complete join (meet) homomorphism is a join (meet respectively) homomorphism. The converse of these are false. In fact a lattice homomorphism need not be a complete meet (or join) homomorphism. For, consider the following . 
Example 4.10. Let $\mathbb{R}$ be the topological space of real numbers with respect to the usual topology. Let $\mathscr{O}(\mathbb{R})$ be the set of all open subsets of $\mathbb{R}$. Then $\mathscr{O}(\mathbb{R})$ together with the inclusion ordering is a complete lattice in which, for any $\left\{A_{\alpha}\right\}_{\alpha \in \Delta} \subseteq \mathscr{O}(\mathbb{R})$,

$$
\sup \left\{A_{\alpha}\right\}_{\alpha \in \Delta}=\bigcup_{\alpha \in \Delta} A_{\alpha} \text { and } \inf \left\{A_{\alpha}\right\}_{\alpha \in \Delta}=\text { The interior of } \bigcap_{\alpha \in \Delta} A_{\alpha} .
$$

Let $\mathscr{P}(\mathbb{R})$ be the complete lattice of all subsets of $\mathbb{R}$ in which the supremums and infimums are simply the set unions and set intersections. Let $i: \mathscr{O}(\mathbb{R}) \longrightarrow$ $\mathscr{P}(\mathbb{R})$ be the inclusion map ( that is, $i(A)=A$ for all $A \in \mathscr{O}(\mathbb{R})$ ). Then clearly $i$ is a lattice homomorphism, since $\mathscr{O}(\mathbb{R})$ is closed under arbitrary unions and finite intersections. Also, for any $\left\{A_{\alpha}\right\}_{\alpha \in \Delta} \subseteq \mathscr{O}(X)$,

$$
i\left(\sup _{\mathscr{O}(\mathbb{R})}\left\{A_{\alpha}\right\}_{\alpha \in \Delta}\right)=i\left(\bigcup_{\alpha \in \Delta} A_{\alpha}\right)=\bigcup_{\alpha \in \Delta} i\left(A_{\alpha}\right)=\sup _{\mathscr{P}(\mathbb{R})}\left\{i\left(A_{\alpha}\right)\right\}_{\alpha \in \Delta}
$$

and therefore $i$ is a complete join homomorphism. However, $i$ is not a complete meet homomorphism, since

$$
\begin{aligned}
i\left(\inf _{\mathscr{O}(\mathbb{R})}\left\{\left(-1, \frac{1}{n}\right)\right\}_{n \in \mathbb{Z}^{+}}\right) & =\text {The interior of } \bigcap_{n \in \mathbb{Z}^{+}}\left(-1, \frac{1}{n}\right) \\
& =\text { The interior of }(-1,0]=(-1,0) \\
\text { and } \inf _{\mathscr{P}(\mathbb{R})}\left\{i\left(-1, \frac{1}{n}\right)\right\}_{n \in \mathbb{Z}^{+}} & =\bigcap_{n \in \mathbb{Z}^{+}}\left(-1, \frac{1}{n}\right)=(-1,0]
\end{aligned}
$$

and hence $i$ is not a complete meet homomorphism.

By considering the dual lattices of the above, we can ascertain that there are complete meet homomorphisms which are not complete join homomorphisms.

Note 4.11. Let $L_{1}$ and $L_{2}$ be complete lattices and $f: L_{1} \longrightarrow L_{2}$ be an order homomorphism. Then, for any subset $A$ of $L_{1}$, we have

$$
f(\sup A) \geq \sup (f(A)) \text { and } f(\inf A) \leq \inf (f(A)) .
$$

From this it follows that $f$ is a complete join homomorphism if and only if

$$
f(\sup A) \leq \sup (f(A)) \text { for all } A \subseteq L_{1}
$$

and that $f$ is a complete meet homomorphism if and only if

$$
f(\inf A) \geq \inf (f(A)) \text { for all } A \subseteq L_{1} .
$$


Let us recall, form Sections 2 and 3, that each of Moore classes, complete lattices and closure operators are in one-to-one correspondence with each other. If $(L, \leq)$ is a complete lattice, then we have the closure space $(L, c)$, where $c$ is the closure operator on $L$ defined by $c(A)=\{x \in L \mid x \leq \sup L\}$ for any $A \subseteq L$. Also, we have the Moore space $(L, \mathscr{M})$ where $\mathscr{M}$ is given by

$\mathscr{M}=\{A \subseteq L \mid c(A)=A\}=\{A \subseteq A \mid x \leq \sup A \Longleftrightarrow x \in A$ for any $x \in L\}$.

Now, we prove the following.

Theorem 4.12. Let $\left(L_{1}, \leq\right)$ and $\left(L_{2}, \leq\right)$ be complete lattices, $\left(L_{1}, c_{1}\right)$ and $\left(L_{2}, c_{2}\right)$ be the corresponding closure spaces and $\left(L_{1}, \mathscr{M}_{1}\right)$ and $\left(L_{2}, \mathscr{M}_{2}\right)$ be the corresponding Moore spaces respectively. Then the following are equivalent to each other for any mapping $f: L_{1} \longrightarrow L_{2}$.

(1). $f$ is a complete join homomorphism of $\left(L_{1}, \leq\right)$ into $\left(L_{2}, \leq\right)$.

(2). $f$ is a homomorphism of the closure space $\left(L_{1}, c_{1}\right)$ into the closure space $\left(L_{2}, c_{2}\right)$.

(3). $f$ is a homomorphism of the Moore space $\left(L_{1}, \mathscr{M}_{1}\right)$ into the Moore space $\left(L_{2}, \mathscr{M}_{2}\right)$

Proof. $(2) \Longleftrightarrow(3)$ is proved in 4.8 .

$(1) \Longrightarrow(2)$ : Suppose that $f$ is a complete join homomorphism. Let $A$ be any subset of $L_{1}$. Then

$x \in c_{1}(A) \Longrightarrow x \leq \sup A \Longrightarrow f(x) \leq f(\sup A)=\sup f(A) \Longrightarrow f(x) \in c_{2}(f(A))$

and hence $f\left(c_{1}(A)\right) \subseteq c_{2}(f(A))$. Thus $f$ is a homomorphism of closure spaces. $(2) \Longrightarrow(1)$ : Suppose that $f$ is a homomorphism of closure spaces. Let $A \subseteq L_{1}$ and $x=\sup A$. Then $x \in c_{1}(A)$

and hence $f(x) \in f\left(c_{1}(A)\right) \subseteq c_{2}(f(A))$, which implies that $f(x) \leq \sup (f(A))$. Therefore

$$
f(\sup A) \leq \sup (f(A)) \text { for all } A \subseteq L_{1} .
$$

Thus $f$ is a complete join homomorphism.

The authors thank Professor U.M. Swamy for his help in preparing this paper. 


\section{References}

[1] G. Birkhoff, Lattice Theory, Amer. Math. Soc., Colloq. Publ. XXV, Providence (1967), U.S.A.

[2] G. Gratzer, General Lattice Theory, Academic Press, New York, Sanfran$\operatorname{sisco}(1978)$.

[3] G.F. Simmons, Introduction to Topology and Modern Analysis, McGrawHill Book Co.Inc, NewYork (1963).

[4] N.B. Stanley, H.P. Sankappanavar, A Course in Universal Algebra, Springer-Verlag, New York (1980).

[5] U.M. Swamy, R.S. Rao, Algebraic topological closure operators, South East Asian Bull. Math., 26 (2002), 669-678. 
